\title{
Prevalence, burden and treatment response of diabetic peripheral neuropathy among attendees of the Diabetic Clinic in the Sri Jayewardenepura General Hospital
}

\author{
Wickramasinghe $\mathbf{T} \mathbf{P}^{1}$, Subasinghe Shyama ${ }^{2}$, Withana A K $\mathbf{G}^{3}$, Wellala D A $\mathbf{H}^{4}$ \\ Journal of the Ceylon College of Physicians, 2016, 47, 20-26
}

\begin{abstract}
Introduction: Diabetic neuropathy is one of the most troublesome complications of diabetes which accounts for substantial morbidity. Thus, early identification and appropriate management of diabetic neuropathy is very important to reduce this burden. This study was done to identify the prevalence of diabetic peripheral neuropathy (DPN) and its impact on the patients' life and to assess the effectiveness of available treatment options.
\end{abstract}

Methodology: 235 patients were screened for symptoms and signs of DPN. An interviewer administered questionnaire was used to assess the impact of neuropathy on their quality of life based on the NeuroQoL questionnaire, and patients were interviewed with regards to treatment efficacy.

Results: $74 \%$ of the screened sample had DPN, of whom $97.1 \%$ were symptomatic. About $40 \%$ of the symptomatic patients complained that DPN affects their quality of life. Seventy four percent mentioned that their symptoms were better with the pharmacological treatment prescribed.

Conclusion: DPN is common among diabetic patients in our clinic. The large majority is symptomatic with their quality of life affected, are reasonably relieved by currently available treatment options.

Key words: diabetic neuropathy, peripheral neuropathy, neuropathy treatment

${ }^{1}$ Demonstrator, Kothalawala Defence University, Sri Lanka.

${ }^{2}$ Consultant Physician, Sri Jayawardenapura General Hospital, Sri Lanka.

${ }^{3}$ Resident House Officer, Sri Jayawardenapura General Hospital, Sri Lanka.

${ }^{4}$ Diabetic Educator Nurse, Sri Jayawardenapura General Hospital, Sri Lanka.

Corresponding author: TPW

E-mail: thilwick87@gmail.com

\section{Introduction}

Longstanding diabetes is associated with complications in vital organs of the body. The disease, particularly when poorly controlled, affects the patient's neurological functions. Many studies have shown that almost $50 \%$ of individuals affected with diabetes are suffering from diabetic neuropathy. ${ }^{1}$ DPN is defined as the presence of symptoms and/or signs of peripheral nerve dysfunction in patients with diabetes after excluding other causes of neurological dysfunction. ${ }^{2}$ According to a review on diabetic polyneuropathy by Dyck et al, DPN is further subdivided into probable and possible DPN based on the presence of specific signs and symptoms. ${ }^{3}$

\section{Objective}

To assess the burden of diabetic peripheral neuropathy (DPN) and response to standard treatment regimens among diabetic clinic attendees in the Sri Jayewardenepura General Hospital in 2015.

\section{Specific objectives}

- $\quad$ To estimate the prevalence of DPN among patients attending the diabetic clinic

- To describe the common clinical presentations of DPN

- To describe how activities of daily life of patients are affected due to DPN

- To assess the effectiveness of available pharmacotherapy on alleviating symptoms of DPN

\section{Methodology}

Study design: A cross-sectional descriptive study was conducted for a period of 15 weeks (15 clinic days) starting from 2nd April 2015. During the study period, a sample of 235 consecutive diabetic patients attending the clinic was selected for the study. Other causes which could lead to peripheral neuropathy were excluded by looking into their habits and past medical, surgical and drug history. Patients with dementia, 
psychiatric disorders, and those unwilling to participate were excluded. To avoid duplicate entries each participant's clinic number was recorded.

Study Instrument: A pre-tes ted interviewer administered questionnaire and a check list were developed to assist the clinician to diagnose DPN. ${ }^{4}$ The douleurneuropathique 4 (DN4) is a screening tool for neuropathic pain consisting of interview questions (DN4-interview) and physical tests. It was validated in Vergata University, Italy and reported a sensitivity of $90 \%$ and specificity of $83 \% .^{5}$ DN4 was translated into Sinhala and Tamil languages to be used in this study, although yet not validated for the Sri Lankan setting as a whole. Tamil patients were interviewed by a Tamil speaking medical officer. Another interviewer administered questionnaire was used to assess the impact of neuropathy on their quality of life. The neuropathy and foot ulcer specific quality of life (NeuroQoL) was the study instrument. ${ }^{6}$ An observation check list was also used to assess the effectiveness of treatment among the same patients.

Method of data collection: Questionnaire was pretested on 40 patients before commencing the proper research, to ensure the validity of the questionnaire. At the end of pretesting, the data collector discussed with the principal investigator and the necessary changes were made. Following pretesting, data were collected by a trained pre-intern medical officer and approximately 20 to 25 patients were interviewed per clinic session. At the first stage of the study, patients were interviewed for symptoms and examined for signs of DPN by the medical officer. All patients were subjected to comprehensive foot examination including the monofilament test, vibration and joint position sensation tests. Pin-prick sensation and light-touch perception were assessed using a $10 \mathrm{~g}$ monofilament. The vibratory threshold was assessed using a $128 \mathrm{~Hz}$ tuning fork. In the second stage of the study, the interviewer administered questionnaire was introduced to the patients to assess the effect of neuropathy on their daily life and the perceived improvement of symptoms for the four pharmacologic treatment modalities (pregabalin, gabapentin, amitriptylin and duloxetine).

Ethical considerations: Approval was obtained from the Ethics Committee of the Sri Jayewardenepura General Hospital. Informed written consent was obtained from all participants before administration of the questionnaire. Patients who refused to participate in the study were allowed to do so without requiring to give reasons for such refusal and they were assured that the services they were receiving from the clinic or hospital would not be affected in whatsoever manner. Confidentiality of the data was ensured and they were informed that the collected data will be used solely for research purposes.
Data analysis: Statistical Package for Social Science (SPSS) version 20 was used for data entry and analysis. Data were cleaned and edited before detailed analysis was carried out. To assess the statistical significance in proportions between different groups Chi Square test and Fisher's exact test were applied, with the level of significance being 0.05 .

\section{Results}

Table 1 describes the age and sex distribution of the study population. The male to female ratio of the study population was 1:1.86 (82: 153) and the majority $(65 \%)$ were female.

Table 1. Demographic data and proportion of DPN among the diabetics ( $\mathrm{N}=\mathbf{2 3 5})$

\begin{tabular}{lcr}
\hline Age (years) & $N$ & $\%$ \\
\hline$<40$ & 22 & 9.4 \\
$41-50$ & 44 & 18.7 \\
$51-60$ & 82 & 34.9 \\
$61-70$ & 67 & 28.5 \\
$>71$ & 20 & 8.5 \\
Sex & & \\
Male & 82 & 34.9 \\
Female & 153 & 65.1 \\
DPN & & \\
Yes & 174 & 74.0 \\
No & 61 & 26.0 \\
\hline
\end{tabular}

Age of the patients ranged from 30 to 80 years with a mean of 56 years. The proportion of DPN with clinical evidence by symptoms and signs among the 235 diabetics recruited was 174 (74\%). Symptoms considered for the diagnosis of DPN were the characteristic symptoms of pain and the presence of current or previous neuropathic ulcers. While sensory deficit and the absence of ankle jerk were the signs considered; of the diagnosed 174 DPN patients, $97.1 \%$ were symptomatic.

Table 2 shows the demographic characteristics and factors associated with DPN among patients with diabetes attending the dedicated clinic. The data show an increasing trend of DPN with age. While $63 \%$ of diabetics below the age of 50 years had DPN, $86 \%$ of affected patients with diabetes above the age of 60 years had DPN, which is statistically significant $(p<0.01)$. There was no significant association of DPN 
with gender $(p>0.05)$. The data also show an association of DPN with the duration of diabetes. Sixty six percent of patients (66\%) with less than 10 years of diabetes and $85 \%$ of diabetics with the duration exceeding 11 years experienced DPN, $(p<0.001)$. Table 3 details the symptoms and signs of patients with DPN. The most common symptoms were an increased sensation of pain $(68.4 \%)$, numbness $(63.8 \%)$ and muscle cramps (63.8\%). In comparison, sensory loss for cold and hot sensation was much less (4.6\%). The commonest signs were dry skin of feet seen in $55.2 \%$ and sensory impairment (loss of protective sensation regarded as the loss of sensation in 8 sites using the $10 \mathrm{~g}$ monofilament test) in $45.4 \%$. Neuropathic ulcers were present in seven and amputations were present in one patient. Table 3 depicts the distribution of neurological symptoms and signs among DPN patients and Table 5 details the social effect of DPN on diabetics. Analysis of the effects of DPN on physical, mental and social wellbeing among diabetics in comparison to diabetics without DPN revealed that depression among DPN patients (17.9\%) was higher than those without DPN (6.6\%), (<0.05). There was a significant difference between DPN and non-DPN diabetic patients in the proportion of adequate sleep, physical exercise and mental stability; which affected a higher proportion of those with DPN. No significant difference was found between these two groups with regard to erectile dysfunction, sexual life, social life, occupation and hobbies.

Of the 174 DPN patients detected by this survey, only $48(27.6 \%)$ were on treatment with specific pharmacotherapy drugs (Table 5). Drugs prescribed were pregabalin, gabapentin, amitriptylin and duloxetine in $25(52.1 \%), 16(33.3 \%), 3(6.3 \%)$ and $4(8.3 \%)$ respectively. The different treatment regimens for individual patient were decided by the treating physician on par with available guidelines. Table 5 shows the reported improvement of symptoms with the differing treatment options, irrespective of the drug regimen and duration of treatment. Among the treated patients 34 (73.9\%) reported an improvement. The improvement with the different drugs is also depicted in Table 6. Since the numbers involved were small, any association between different drugs and improvement status could not be statistically tested. The proportion that reported an improvement within 6 months and beyond 6 months of therapy were 73.7 and 76.0 and not significantly different.

Table 2. Factors associated with DPN

\begin{tabular}{|c|c|c|c|c|c|}
\hline \multirow{3}{*}{ Characteristic } & \multicolumn{4}{|c|}{ DPN Status } & \multirow[t]{3}{*}{$p^{*}$} \\
\hline & \multicolumn{2}{|c|}{ Yes $(N=174)$} & \multicolumn{2}{|c|}{ No $(N=61)$} & \\
\hline & $N$ & $\%$ & $N$ & $\%$ & \\
\hline \multicolumn{6}{|l|}{ Age (years) } \\
\hline$=<50$ & 42 & 63.6 & 24 & 36.4 & $<0.01$ \\
\hline $51-60$ & 57 & 69.5 & 25 & 30.5 & \\
\hline$=>60$ & 75 & 86.2 & 12 & 13.8 & \\
\hline \multicolumn{6}{|l|}{ Sex } \\
\hline Male & 57 & 69.5 & 25 & 30.5 & $>0.05$ \\
\hline Female & 117 & 76.5 & 36 & 23.5 & \\
\hline \multicolumn{6}{|c|}{ Duration of DM (Years) } \\
\hline$=<10$ & 101 & 66.4 & 51 & 33.6 & $<0.01$ \\
\hline $11-20$ & 50 & 89.3 & 6 & 10.7 & \\
\hline$=>20$ & 23 & 85.2 & 4 & 14.8 & \\
\hline
\end{tabular}

* From Chi square test 
Table 3. Distribution of neurological symptoms and signs among patients with DPN

\begin{tabular}{lll}
\hline Symptoms and signs & $N$ & N
\end{tabular}

\section{Symptoms}

Numbness

Pricking pain

Burning pain

Tingling

Pain while cloth touching the legs

Cannot feel the cold sensation

Cannot feel the hot sensation

Muscle cramps

63.8

Increased sensation

Altered sensation

119

\section{Signs}

Dry feet

Cracked feet 33.3

Pulse

Weak

11.5

Bounding

Hair loss

Shiny skin

Deformities

Charcot joints

Absent

AJL

Diminished

\section{AJR}

Absent

6.3

\section{Diminished}

10.9

Sensory impairment

Total

Vibration

Right

Left

JPS

Right

Left

Previous ulcers

9

Current ulcers

7

AJL - ankle jerk left side; AJR - ankle jerk reflex right side; JPS - joint position sense 
Table 4. The effect of DPN on social wellbeing

\begin{tabular}{|c|c|c|c|c|c|}
\hline \multirow[t]{3}{*}{ Characteristic } & \multicolumn{4}{|c|}{ DPN Status } & \multirow[t]{3}{*}{$p^{x}$} \\
\hline & \multicolumn{2}{|c|}{ Yes $(N=174)$} & \multicolumn{2}{|c|}{ No $(N=61)$} & \\
\hline & $N$ & $\%$ & N & $\%$ & \\
\hline \multicolumn{6}{|l|}{ Depression } \\
\hline Yes & 31 & 17.9 & 4 & 6.6 & $<0.05$ \\
\hline No & 142 & 82.1 & 57 & 93.4 & \\
\hline \multicolumn{6}{|c|}{ Erectile dysfunction ${ }^{\star \star}$} \\
\hline Yes & 16 & 29.1 & 3 & 12.0 & $>0.05$ \\
\hline No & 39 & 70.9 & 22 & 88.0 & \\
\hline \multicolumn{6}{|l|}{ Sleep affected } \\
\hline Yes & 76 & 43.7 & 12 & 19.7 & $<0.01$ \\
\hline No & 98 & 56.3 & 49 & 80.3 & \\
\hline \multicolumn{6}{|c|}{ Social life affected } \\
\hline Yes & 23 & 13.4 & 5 & 8.2 & $>0.05$ \\
\hline No & 149 & 86.6 & 56 & 91.8 & \\
\hline \multicolumn{6}{|c|}{ Hobbies affected } \\
\hline Yes & 36 & 20.8 & 7 & 11.5 & $>0.05$ \\
\hline No & 137 & 79.2 & 54 & 88.5 & \\
\hline \multicolumn{6}{|c|}{ Sex life affected } \\
\hline Yes & 22 & 13.3 & 3 & 5.0 & $>0.05$ \\
\hline No & 143 & 86.7 & 57 & 95.0 & \\
\hline \multicolumn{6}{|c|}{ Exercise affected } \\
\hline Yes & 60 & 35.3 & 9 & 14.8 & $<0.01$ \\
\hline No & 110 & 64.7 & 52 & 85.2 & \\
\hline \multicolumn{6}{|c|}{ Occupation affected } \\
\hline Yes & 28 & 17.4 & 7 & 11.9 & $>0.05$ \\
\hline No & 133 & 82.6 & 52 & 88.1 & \\
\hline \multicolumn{6}{|c|}{ Mental stability affected } \\
\hline Yes & 50 & 28.7 & 9 & 14.8 & $<0.05$ \\
\hline No & 124 & 71.3 & 52 & 85.2 & \\
\hline
\end{tabular}

* From Chi square test, ${ }^{* *}$ Out of males who responded $(n=80)$

Table 5. Distribution of specific treatment given for DPN and degree of symptom improvement reported

\begin{tabular}{lrr}
\hline Treatment & $n$ & $\%$ \\
\hline Treatment status $(\mathrm{n}=174)$ & & \\
$\quad$ Treated & 48 & 27.6 \\
$\quad$ Not treated & 126 & 72.4 \\
Drugs given $(\mathrm{n}=48)$ & & 52.1 \\
$\quad$ Pregabalin & 25 & Continued
\end{tabular}




$\begin{array}{lrr}\text { Gabapentin } & 16 & 33.3 \\ \text { Amitriptylin } & 3 & 6.3 \\ \text { Duloxetine } & 4 & 8.3 \\ & & \\ \text { Improvement reported } & & 73.9 \\ \quad \text { Yes } & 34 & 26.1 \\ \quad \text { No } & 12 & \end{array}$

\begin{tabular}{|c|c|c|c|c|c|}
\hline & \multicolumn{5}{|c|}{ Improvement } \\
\hline & & & & & \\
\hline & $n$ & $\%$ & $n$ & $\%$ & $n$ \\
\hline Pregabalin & 18 & 75.0 & 6 & 25.0 & 24 \\
\hline Gabapentin & 11 & 68.8 & 5 & 31.3 & 16 \\
\hline Amitriptylin & 2 & 68.7 & 1 & 33.3 & 3 \\
\hline Duloxetine & 3 & & 0 & & 3 \\
\hline Total & 34 & & 12 & & 46 \\
\hline
\end{tabular}

Table 6. Reported symptomatic improvement based on the duration of treatment

\begin{tabular}{|c|c|c|c|c|c|}
\hline \multirow[t]{3}{*}{ Improvement } & \multicolumn{4}{|c|}{ Duration of treatment } & \multirow[t]{3}{*}{$p^{*}$} \\
\hline & \multicolumn{2}{|c|}{$<=6$ months } & \multicolumn{2}{|c|}{$>6$ months } & \\
\hline & $N$ & $\%$ & $N$ & $\%$ & \\
\hline Improved & 14 & 73.7 & 19 & 76.0 & $>0.05^{*}$ \\
\hline Not improved & 5 & 26.3 & 6 & 24.0 & \\
\hline
\end{tabular}

* From Fisher's Exact Test

\section{Discussion}

The prevalence of DPN in newly diagnosed patient with diabetes is estimated to be $8 \%$ and greater than $50 \%$ in patient with long standing disease. ${ }^{7}$ In our study, the prevalence of DPN was $74 \%$, of whom $5.2 \%$ had a duration of diabetes less than one year. Thirty four percent had diabetes for less than five years. These relatively higher values may be due to ethnic differences of the study sample. Yet another possibility is that these patients had undiagnosed and untreated diabetes for a long period. Therefore we should aim to strengthen our screening programmes, of at least among high risk population, and in particular ensure improved glycaemic control within the first five years since the detection of diabetes. A previous Sri Lankan study carried out in the community reported that the prevalence of DPN was much lower than our finding. ${ }^{7}$ This difference may be due to the fact that our study is confined to a tertiary hospital based clinic. Several epidemiological studies have shown that the duration and severity of diabetes (assessed by glycated haemoglobin) are significant risk factors for the development of DPN. ${ }^{9}$ Our study also showed that the duration of diabetes is a significant risk factor.

Symptoms such as burning, tingling, numbness, shooting (electric shock) and stabbing sensations are present in 33\% of patients with DPN according to some studies. ${ }^{10,11}$ Our study found that $63.8 \%$ complained of numbness, $34.5 \%$ complained of burning pain and $42.5 \%$ of patients complained of a pricking type of pain in their soles. We found that $68.4 \%$ of the cohort 
with DPN complained that they have increased sensation in the affected area and that $63.8 \%$ had frequent muscle cramps. These symptoms are most often worst at night and can disrupt sleep. ${ }^{12}$ We also detected that $43.7 \%$ of patients had sleep disturbances due to their neuropathic symptoms.

Although there is no treatment currently available to repair the nerve damage of DPN, there is effective symptomatic treatment available for the relief of symptoms associated with DPN. Several randomized placebo controlled trials have shown the effectiveness of tricyclic anti-depressants in the treatment of painful diabetic neuropathy. ${ }^{13,14}$ Serotonin - noradrenaline reuptake inhibitors have also been shown to be effective for painful diabetic neuropathy, e.g. venlafaxine and duloxetine. ${ }^{15,16}$. In an eight week, multicenter randomized controlled trial of 165 patients with DPN, gabapentin was reported to give moderate pain relief when compared with placebo. ${ }^{17}$ In a pooled analysis from 7 randomized, placebo-controlled trials pregabalin was shown to relieve pain and improve the quality of life, measures of social functioning and mental health as well as to improve sleep. ${ }^{18}$

The affected patients who received such treatment in this study also reported a good response to them. Three quarters of the affected group responded to the treatment prescribed. Patients prescribed pregabalin, reported a good response in $78.26 \%$ while $68.75 \%$ of the group prescribed gabapentin said that they had satisfactory response.

\section{Conclusion}

The prevalence of diabetic peripheral neuropathy is high in this study. Symptomatic DPN can affect the life of the person to a considerable degree. The currently available treatment give good symptomatic relief. Regular screening programmes are recommended to strengthen early detection of undiagnosed cases with a view to prevention of such complications of diabetes.

\section{Acknowledgments}

We express our gratitude to patients of the Diabetes Clinic for their ready participation to share their personal information for this study. We thank the medical officers and staff of the Medical Clinic, particularly Jayanthi, Viraj and Swarna for their ready support.

\section{References}

1. Tesfaye S, Selvarajah D. Advances in the epidemiology, pathogenesis and management of diabetic peripheral neuropathy. Diabetes Metab Res Rev. 2012; 28 (supplement 1): 8-14.

2. Boulton AJM. Management of Diabetic Peripheral Neuropathy. Clinical Diabetes. 2005; 23(1): 9-15.
3. Peter J Dyck, Solomon T, et al. Diabetic neuropathies: update on definitions, diagnostic criteria, estimation of severity, and treatments, Diabetes Metab Res Rev 2011; 27: $620-8$.

4. Deli G, Bosnyak E, Pusch G, Komoly S, Feher G. Diabetic Neuropathies: Diagnosis and Management. Neuro Endocrinology 2014; 98(4): 267-80.

5. Spallone V, Morganti R, D'Amato C, Greco C, Cacciotti L, Marfia GA. Validation of DN4 as a screening tool for neuropathic pain in painful diabetic polyneuropathy. Diabetic Medicine 2012; 29(5): 578-85.

6. Vileikyte L, Peyrot M, Bundy C, Rubin RR, Leventhal H, Mora $P$, et al. The development and validation of a neuropathy and foot ulcer-specific quality of life instrument. Diabetes Care 2003; 26(9): 2549-55.

7. Boulton AJ, VinikAl, Arezzo JC, Bril V, Feldman EL, Freeman R, Malik RA, Maser RE, Sosenko JM, Ziegler D. Diabetic neuropathies: a statement by the American Diabetes Association. Diabetes Care 2005; 28(4): 956-62.

8. Katulanda $P$, Ranasinghe $P$, Jayawardene R. Constantine GR, Sheriff MHR, Matthews DR. The prevealnce, patterns and predictors of DPN in a developing country. Diabetology and Metabolic Syndrome 2012; 4: 21.

9. Tesfaye S, Stevens LK, Stephenson JM, Fuller JH, Plater M, Ionescu-Tirgoviste $\mathrm{C}$, et al. Prevalence of diabetic peripheral neuropathy and its relation to glycaemic control and potential risk factors: the EURODIAB IDDM Complications Study. Diabetologia 1996; 39(11): 1377-84.

10. Tesfaye S, Boulton AJ, Dyck PJ, et al. Diabetic neuropathies: update on definitions, diagnostic criteria, estimation of severity and treatments. Diabetes Care 2010; 33(10): 2285-93.

11. Davies M, Broply S, Willianus R, et al. The prevalence, severity and impact of painful DPN in type $2 \mathrm{DM}$. Diabetes Care 2005; 29(7): 1518-22.

12. Quattrini C, Tesfaye S. Understanding the impact of painful diabetic neuropathy. Diabetes Metab Res Rev 2003; 19 (Suppl 1): S2-8.

13. Max MB, Lynch SA, Muir J, et al. Effects of desipramine, amitriptyline and fluoxetine on pain in diabetic neuropathy. N Engl J Med 1992; 326(19):1250-6.

14. Kvinesdal B, Molin J, Froland A, et al. Imipramine treatment of painful diabetic neuropathy. JAMA 1984; 251(13): 1727-30.

15. Simpson DA. Gabapentin and Venlafaxine for the treatment of painful diabetic neuropathy. $\mathrm{J}$ Clin Neuromuscular Dis 2001; 3(2): 53-62.

16. Goldstein DJ, Lu Y, Detke MJ, Lee TC, lyengar S. Duloxetine vs. placebo in patients with painful diabetic neuropathy. Pain 2005; 116(1-2): 109-18.

17. Backonja M, Beydoun A, Edwards KR, et al. Gabapentin for the symptomatic treatment of painful neuropathy in patients with DM: a randomized controlled trial. JAMA 1998; 280(21): 1831-6.

18. Freeman R, Durso-Decrz E, Emir B. Efficacy, safety and tolerability of pregabalin treatment for painful DPN: findings from screen randomized, controlled trials across a range of dose. Diabetic Care 2008; 31(7): 1448-54. 